https://doi.org/10.15407/ujpe63.9.777

M.V. VAVRUKH, D.V. DZIKOVSKYI, S.V. SMERECHYNSKYI

Ivan Franko National University of Lviv, Department of Astrophysics

(8, Kyrylo and Methodiy Str., Lviv 79005, Ukraine; e-mail: mvavrukh@gmail.com)

\title{
CONSIDERATION OF THE COMPETING FACTORS IN CALCULATIONS OF THE CHARACTERISTICS OF NON-MAGNETIC DEGENERATE DWARFS
}

\begin{abstract}
Using the equation of state of the electron-nuclear model at high densities and the mechanical equilibrium equation, we have investigated the influence of interparticle interactions and the axial rotation on the macroscopic characteristics (mass, surface shape) of massive degenerate dwarfs. We propose a method of solving the equilibrium equation in the case of rotation that uses the basis of universal functions of the radial variable. The conditions, under which the axial rotation can compensate for a weight loss of the mass due to the Coulomb interactions, have been established. The maximal value of the relativistic parameter, at which the stability is disturbed, is determined within the general theory of relativity (GTR).
\end{abstract}

Ke ywords: mechanical equilibrium equation, interparticle interactions, axial rotation, basis of universal functions, stability of dwarfs.

\section{Introduction}

The discovery of the degenerate dwarfs at the beginning of the XXth century [1] gave rise to the problem of existence and stability of stars, which have no sources of energy. R. Fowler's idea [2] that the existence of these objects is due to the pressure of the degenerate electron gas at high densities of matter led to the formation of an electron-nuclear model, in which a star consists of an ideal degenerate relativistic electron subsystem in the paramagnetic state at $T=0 \mathrm{~K}$ and a static nuclear subsystem, which is considered as a continuous classical environment $[3,4]$. In the frame of this model, the theory of cold degenerate dwarfs was constructed by S. Chandrasekhar, the main results of which are restrictions on the mass $\left(M \leq 1.45 M_{\odot}\right)$ and the peculiar "mass-radius" ratio. In the works by E. Shatzman [5], S. Kaplan [6], R. James [7], E. Salpeter [8], Ya. Zeldovich and I. No-

(C) M.V. VAVRUKH, D.V. DZIKOVSKYI,

S.V. SMERECHYNSKYI, 2018

ISSN 2071-0194. Ukr. J. Phys. 2018. Vol. 63, No. 9 vikov [9], S. Shapiro and S. Teukolsky [10], and other researchers, some generalizations of Chandrasekar's model, which consider important factors of the formation of a structure of degenerate dwarfs, were proposed.

The development of the theory was restrained by the lack of observable data. The situation changed at the end of the XXth century, when a large amount of spectral data on the degenerate dwarfs in a vicinity of the Sun was obtained with help of the space observatories. It turned out that the degenerate dwarfs are characterized by the same variety of characteristics like stars of other types. The most striking fact indicating the limitation of applying Chandrasekhar's model is the distribution of dwarfs with small and medium masses on the "mass-radius" plane [11], which is a manifestation of the incomplete degeneration of the subsystem of electrons, since the effective temperatures of the photosphere of some dwarfs reach $10^{5} \mathrm{~K}$. However, the influence of temperature effects on the characteristics of massive dwarfs is very 
small. In the case of massive non-magnetic dwarfs, the main factors of the structure formation are interparticle Coulomb interactions and the axial rotation, which are competing ones. The dwarfs with masses approaching Chandrasekar's limit are found in the binary systems, where the important factor at certain stages of evolution is the exchange of mass between the components.

The detailed calculations of the degenerate dwarf characteristics, when various factors of the formation of these objects are simultaneously taken into account, are relevant in connection with the problem of the stability of the degenerate dwarfs, as well as with the hypothesis that massive degenerate dwarfs are precursors of supernovae of type Ia.

The main purpose of our work is to set restrictions on the mass of dwarfs, while considering the Coulomb interactions and the axial rotation.

\section{Equilibrium Equation}

The internal structure of a star with axial rotation is determined by the equilibrium equation [12]: at each point given by the radius vector $\mathbf{r}$, the condition

$\nabla P(\mathbf{r})=-\rho(\mathbf{r}) \nabla\left\{\Phi_{\text {grav }}(\mathbf{r})+\Phi_{c}(\mathbf{r})\right\}$

is satisfied, where $P(\mathbf{r})$ is the local pressure, $\rho(\mathbf{r})$ is the local density of matter,

$\Phi_{\text {grav }}(\mathbf{r})=-G \int \frac{\rho\left(\mathbf{r}^{\prime}\right) d \mathbf{r}^{\prime}}{\left|\mathbf{r}-\mathbf{r}^{\prime}\right|}$

determines the gravity potential, and $\Phi_{c}(\mathbf{r})$ is the centrifugal one. We consider that the distribution of matter has the axial symmetry, and the axis of rotation passes through the center of mass of the star. In the spherical coordinate system, the coordinate origin being at the center of mass,

$\Phi_{c}(\mathbf{r})=-\frac{1}{2} \omega^{2} r^{2} \sin ^{2} \theta$

where $\omega$ is the constant angular velocity, and $\theta$ is the polar angle. The density of matter $\rho(\mathbf{r})$ is expressed in terms of the local value of the relativistic parameter

$x(\mathbf{r})=\frac{\hbar}{m_{0} c}\left(3 \pi^{2} n(\mathbf{r})\right)^{1 / 3}$,

$m_{0}$ is the electron mass, $c$ is the speed of light, and $n(\mathbf{r})$ is the number density of electrons at the point r. According to definition (4),

$\rho(\mathbf{r})=m_{u} \mu_{e} n(\mathbf{r})=\frac{m_{u} \mu_{e}}{3 \pi^{2}} x^{3}(\mathbf{r})\left(\frac{m_{0} c}{\hbar}\right)^{3}$,

where $m_{u}$ is the atomic mass unit, $\mu_{e}=A / z$ ( $A$ is the mass number, $z$ is the charge of a nucleus).

The equation of state of the macroscopic homogeneous electron-nuclear model at $T=0 \mathrm{~K}$ was obtained in work [13]. This equation is convenient to present in the form

$P(x)=\frac{\pi m_{0}^{4} c^{5}}{3 h^{3}}\{\mathcal{F}(x)-f(x \mid z)\}$,

where $\mathcal{F}(x)$ is the contribution of the degenerate ideal relativistic electron gas, and $f(x \mid z)>0$ is the contribution of interactions (see Appendix 1). To describe the star, we have used expression (6) in the local approximation by replacing $x$ with its local value $x(\mathbf{r})$. In this approximation, the equilibrium equation is reduced to such differential equation for the local value of the relativistic parameter:

$\Delta\left\{\left[1+x^{2}(\mathbf{r})\right]^{1 / 2}-1\right\}=2 \frac{\omega^{2} m_{u} \mu_{e}}{m_{0} c^{2}}-$

$-\frac{32 \pi^{2} G}{3(h c)^{3}}\left(m_{u} \mu_{e} m_{0} c^{2}\right) x^{3}(\mathbf{r})+$

$\left.+(2 x(\mathbf{r}))^{-3}\left(\frac{d}{d x(\mathbf{r})} f(x(\mathbf{r})) \mid z\right)\right) \Delta x(\mathbf{r})+$

$+2^{-3}\left(\frac{d}{d x(\mathbf{r})}\left[\frac{1}{x^{3}(\mathbf{r})} \frac{d}{d x(\mathbf{r})} f(x(\mathbf{r}) \mid z)\right]\right)(\nabla x(\mathbf{r}))^{2}$.

Here, $\Delta$ is the Laplace operator in the variables $(r, \theta)$. The derivatives from Eq. (7) are illustrated in Fig. 1.

Let us introduce the dimensionless variables

$\xi=\frac{r}{\lambda}, \quad Y(\xi, \theta)=\varepsilon_{0}^{-1}\left\{\left[1+x^{2}(r)\right]^{1 / 2}-1\right\}$,

as well as the dimensionless angular velocity according to the expression

$\Omega^{2}=2 \frac{\omega^{2} m_{u} \mu_{e} \lambda^{2}}{m_{0} c^{2} \varepsilon_{0}}$

where

$\varepsilon_{0} \equiv \varepsilon_{0}\left(x_{0}\right)=\left[1+x_{0}^{2}\right]^{1 / 2}-1, \quad x_{0} \equiv x(0)$.

ISSN 2071-0194. Ukr. J. Phys. 2018. Vol. 63, No. 9 
If the scale $\lambda$ is determined by the expression

$$
\frac{32 \pi^{2} G}{3(h c)^{3}}\left(m_{u} \mu_{e} m_{0} c^{2} \lambda \varepsilon_{0}\right)^{2}=1,
$$

then the equilibrium equation has the following dimensionless form:

$$
\begin{aligned}
& \Delta(\xi, \theta) Y(\xi, \theta)=\Omega^{2}-\Gamma^{3}(\xi, \theta)+ \\
& +\varphi_{1}(\xi, \theta \mid z) \Delta(\xi, \theta) \Gamma(\xi, \theta)+ \\
& +\varphi_{2}(\xi, \theta \mid z)\left\{\left[\frac{\partial}{\partial \xi} \Gamma(\xi, \theta)\right]^{2}+\frac{1-t^{2}}{\xi^{2}}\left[\frac{\partial}{\partial t} \Gamma(\xi, \theta)\right]^{2}\right\} .
\end{aligned}
$$

Here, the notations are as follows:

$$
\begin{aligned}
& \Gamma(\xi, \theta)=\left[Y^{2}(\xi, \theta)+\frac{2}{\varepsilon_{0}} Y(\xi, \theta)\right]^{1 / 2} ; \\
& \varphi_{1}(\xi, \theta \mid z)=\left.(2 x)^{-3} \frac{d f(x \mid z)}{d x}\right|_{x=x(\xi, \theta)} \text {; } \\
& \varphi_{2}(\xi, \theta \mid z)=\left.\frac{\varepsilon_{0}}{8} \frac{d}{d x}\left\{\frac{1}{x^{3}} \frac{d f(x \mid z)}{d x}\right\}\right|_{x=x(\xi, \theta)} ; \\
& x(\xi, \theta)=\varepsilon_{0} \Gamma(\xi, \theta) ; \quad t=\cos \theta ; \\
& \Delta(\xi, \theta)=\Delta_{\xi}+\frac{1}{\xi^{2}} \Delta_{\theta} ; \quad \Delta_{\xi}=\frac{1}{\xi^{2}} \frac{\partial}{\partial \xi}\left(\xi^{2} \frac{\partial}{\partial \xi}\right) ; \\
& \Delta_{\theta}=\frac{\partial}{\partial t}\left(1-t^{2}\right) \frac{\partial}{\partial t} \text {. }
\end{aligned}
$$

In Eq. (12), the independent parameters $x_{0}, \Omega^{2}, z$ appear. The equation in partial derivatives (12) satisfies the boundary conditions

$Y(0, \theta)=1 ; \quad \frac{\partial}{\partial \xi} Y(\xi, \theta)=0 \quad$ by $\quad \xi=0$.

In order to highlight the analytical dependence on model parameters, we introduce an approximate solution of Eq. (12). Let us consider Eq. (12) without rotation, by setting $\Omega=0$ and replacing $Y(\xi, \theta)$ with the function $y(\xi)$ according to the spherical symmetry of the problem.

\section{Influence of the Interactions}

The function $y(\xi)$ satisfies the one-dimensional differential equation

$\Delta_{\xi} y(\xi)=-\left\{y^{2}(\xi)+\frac{2}{\varepsilon_{0}} y(\xi)\right\}^{3 / 2}+$

ISSN 2071-0194. Ukr. J. Phys. 2018. Vol. 63, No. 9
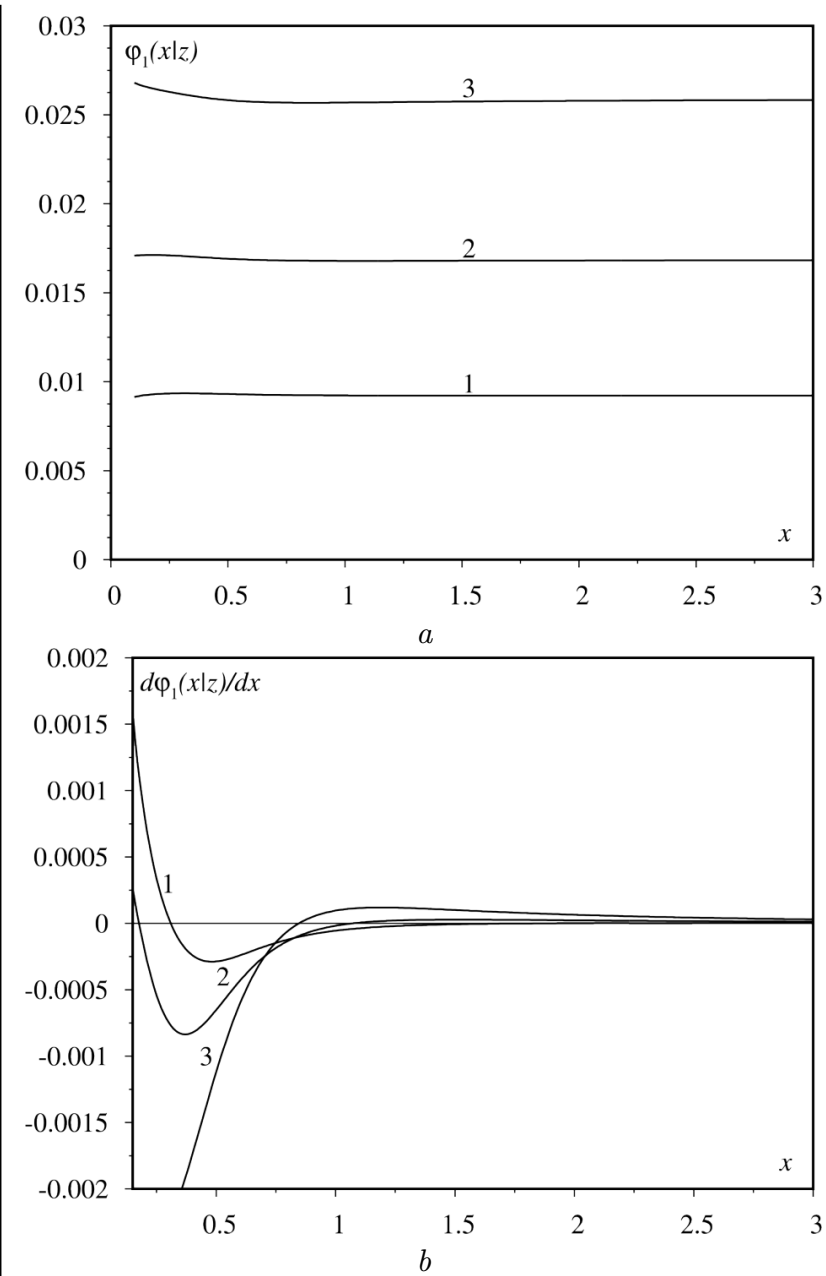

Fig. 1. Dependence of the functions $\varphi_{1}(x \mid z)=(2 x(r))^{-3} \times$ $\times d f(x(r)) \mid z) / d x(r)(a)$ and $d \varphi_{1}(x \mid z) / d x(b)$ on the relativistic parameter $x$ and the nuclear charge $z$ (curve $1-z=2 ; 2-$ $z=6 ; 3-z=12$ )

$$
\begin{aligned}
& +\varphi_{1}(\xi \mid z) \Delta_{\xi}\left[y^{2}(\xi)+\frac{2}{\varepsilon_{0}} y(\xi)\right]^{1 / 2}+ \\
& +\varphi_{2}(\xi \mid z)\left\{\frac{d}{d \xi}\left[y^{2}+\frac{2}{\varepsilon_{0}} y(\xi)\right]^{1 / 2}\right\}^{2}
\end{aligned}
$$

in which $x_{0}$ and $z$ are the parameters, and the functions $\varphi_{i}(\xi \mid z)$ are determined by Eqs. (13), where one should make a replacement $x \rightarrow \varepsilon_{0}\left(y^{2}(\xi)+\right.$ $\left.+\frac{2}{\varepsilon_{0}} y(\xi)\right)^{1 / 2}$. Regular solutions of the equation satisfy the conditions $y(0)=1, y^{\prime}(0)=0$. The condition $y(\xi)=0$ determines the dimensionless radius of the star $\xi_{1}\left(x_{0} \mid z\right)$. Setting $\varphi_{1}=\varphi_{2}=0$, the equation becomes a one-parameter equilibrium equation of Chandrasekar's model (the standard model). The 
Table 1. Dependence of the dimensionless radius $\xi_{1}\left(x_{0} \mid z\right)$ on the parameters $x_{0}$ and $z\left(\xi_{1}\left(x_{0}\right)\right.$ corresponds to the standard model)

\begin{tabular}{|r|c|c|c|c|c|}
\hline & & \multicolumn{4}{|c|}{$\xi_{1}\left(x_{0} \mid z\right)$} \\
\cline { 3 - 6 }$x_{0}$ & $\xi_{1}\left(x_{0}\right)$ & & \multicolumn{4}{|c|}{} \\
\cline { 3 - 6 } & & $z=2$ & $z=6$ & $z=12$ & $z=26$ \\
\hline & & & & & \\
2.0 & 1.03401 & 1.00101 & 0.98801 & 0.97401 & 0.94801 \\
3.0 & 2.78201 & 2.74601 & 2.72401 & 2.70001 & 2.65601 \\
4.0 & 3.30701 & 3.27001 & 3.24701 & 3.22001 & 3.17201 \\
5.0 & 3.70701 & 3.67001 & 3.64501 & 3.61701 & 3.56601 \\
6.0 & 4.02301 & 3.98601 & 3.96001 & 3.93101 & 3.87801 \\
7.0 & 4.28001 & 4.24301 & 4.21701 & 4.18701 & 4.13201 \\
8.0 & 4.49301 & 4.45601 & 4.43001 & 4.39901 & 4.34401 \\
9.0 & 4.67401 & 4.63701 & 4.61001 & 4.57901 & 4.52201 \\
10.0 & 4.82801 & 4.79101 & 4.76401 & 4.73301 & 4.67601 \\
15.0 & 5.35801 & 5.32201 & 5.29401 & 5.26301 & 5.20301 \\
20.0 & 5.67001 & 5.63501 & 5.60701 & 5.57501 & 5.51501 \\
25.0 & 5.87701 & 5.84201 & 5.81401 & 5.78201 & 5.72201 \\
30.0 & 6.02401 & 5.98901 & 5.96101 & 5.92901 & 5.86901 \\
\hline
\end{tabular}

Table 2. Dependence of the dimensionless mass $\mathcal{M}\left(x_{0} \mid z\right)$ on the parameters $x_{0}, z\left(\mathcal{M}\left(x_{0}\right)\right.$ corresponds to the standard model)

\begin{tabular}{|c|c|c|c|c|c|}
\hline \multirow{2}{*}{$x_{0}$} & \multirow{2}{*}{$\mathcal{M}\left(x_{0}\right)$} & \multicolumn{4}{|c|}{$\mathcal{M}\left(x_{0} \mid z\right)$} \\
\hline & & $z=2$ & $z=6$ & $z=12$ & $z=26$ \\
\hline 1.0 & 0.707066 & 0.689037 & 0.673304 & 0.65581 & 0.624491 \\
\hline 2.0 & 1.24303 & 1.22092 & 1.20126 & 1.17904 & 1.13834 \\
\hline 3.0 & 1.51862 & 1.49465 & 1.47331 & 1.44912 & 1.4045 \\
\hline 4.0 & 1.67141 & 1.64646 & 1.62426 & 1.59907 & 1.55247 \\
\hline 5.0 & 1.76395 & 1.73843 & 1.71573 & 1.68996 & 1.64222 \\
\hline 6.0 & 1.82404 & 1.79816 & 1.77515 & 1.74901 & 1.70056 \\
\hline 7.0 & 1.86521 & 1.83909 & 1.81586 & 1.78948 & 1.74054 \\
\hline 8.0 & 1.89462 & 1.86832 & 1.84495 & 1.81839 & 1.76911 \\
\hline 9.0 & 1.91634 & 1.88992 & 1.86645 & 1.83976 & 1.79023 \\
\hline 10.0 & 1.93284 & 1.90633 & 1.88277 & 1.85599 & 1.80626 \\
\hline 15.0 & 1.97619 & 1.94943 & 1.92567 & 1.89863 & 1.84839 \\
\hline 20.0 & 1.99337 & 1.96651 & 1.94268 & 1.91554 & 1.86508 \\
\hline 25.0 & 2.00186 & 1.97495 & 1.95108 & 1.92389 & 1.87331 \\
\hline 30.0 & 2.00665 & 1.97972 & 1.95583 & 1.92861 & 1.87795 \\
\hline
\end{tabular}

solutions of Eq. (15) were found numerically. The dependence $\xi_{1}\left(x_{0} \mid z\right)$ on the parameters $x_{0}$ and $z$ is shown in Table 1, where the dimensionless radius of the star in the standard model $\xi_{1}\left(x_{0}\right)$ is given for comparison. As can be seen from this table, account- ing for interactions leads to a decrease of the radius $\left\{\xi_{1}\left(x_{0}\right)-\xi_{1}\left(x_{0} \mid z\right)\right\}\left(\xi_{1}\left(x_{0}\right)\right)^{-1}$ by $0.7 \%$ at $z=2,1.2 \%$ at $z=6,1.85 \%$ at $z=12$ and $3 \%$ at $z=26$.

The mass and radius of the star are determined by the expressions

$$
\begin{aligned}
& M\left(x_{0}\left|\mu_{e}\right| z\right)=\frac{M_{0}}{\mu_{e}^{2}} \mathcal{M}\left(x_{0} \mid z\right), \\
& \mathcal{M}\left(x_{0} \mid z\right)=\int_{0}^{\xi_{1}\left(x_{0} \mid z\right)} \xi^{2}\left\{y^{2}(\xi)+\frac{2}{\varepsilon_{0}} y(\xi)\right\}^{3 / 2} d \xi \\
& R\left(x_{0}\left|\mu_{e}\right| z\right)=\frac{R_{0}}{\mu_{e}} \frac{\xi_{1}\left(x_{0} \mid z\right)}{\varepsilon_{0}}
\end{aligned}
$$

where the mass and radius scale are the combinations of the universal constants

$$
\begin{aligned}
& M_{0}=\left(\frac{3}{2}\right)^{1 / 2} \frac{1}{4 \pi}\left(\frac{h c}{G m_{u}^{2}}\right)^{3 / 2} m_{u}= \\
& =5.740247 \times 10^{33} \mathrm{~g} \approx 2.88695 M_{\odot} ; \\
& R_{0}=\left(\frac{3}{2}\right)^{1 / 2} \frac{1}{4 \pi}\left(\frac{h^{3}}{c G}\right)^{1 / 2} \frac{1}{m_{0} m_{u}}= \\
& =0.776885 \times 10^{9} \mathrm{~cm} \approx 1.11623 \times 10^{-2} R_{\odot} .
\end{aligned}
$$

The dependence $\mathcal{M}\left(x_{0} \mid z\right)$ on the parameters $x_{0}$ and $z$ and the dimensionless mass $\mathcal{M}\left(x_{0}\right)$ in the standard model are given in Table 2 . The relative decrease of the mass caused by the influence of interactions $\left\{\mathcal{M}\left(x_{0}\right)-\mathcal{M}\left(x_{0} \mid z\right)\right\}\left(\mathcal{M}\left(x_{0}\right)\right)^{-1}$ is approximately $1.4 \%$ at $z=2,2.7 \%$ at $z=6,4.1 \%$ at $z=12$, and $7 \%$ at $z=26$ in the region $x_{0} \geq 10$ (see Table 2 ).

For intermediate and large values of the relativistic parameter, the function $f(x \mid z)$ is approximately proportional to $x^{4}$. In other words, the expression $x^{-3} d f / d x$ is close to the constant value, and its derivative with respect to $x$ is very small. This gives the opportunity to get an approximate estimate of dwarf's characteristics without solving Eq. (15) numerically. Due to the fact that, in the core of a massive dwarf, $x(r)$ is very close to $x_{0}$, the expression $x^{-3}(r) d f(x(r)) / d x(r)$ can be replaced by the $\varphi_{1}\left(x_{0} \mid z\right)=d f\left(x_{0} \mid z\right) / d x_{0}$. One can neglect the term proportional to $(\nabla x(r))^{2}$ and introduce a new dimensionless coordinate $\tilde{\xi}=\xi / k$ at $k=\left\{1-\varphi_{1}\left(x_{0} \mid z\right)\right\}^{1 / 2}$. In this case, Eq. (15) can be reduced to the equation of the standard model. In this approximation, the solution of Eq. (15) is $y(\xi)=\tilde{y}(\xi / k)$, where $\tilde{y}(\xi)$ is a 
solution of the equation in the standard model

$$
\begin{aligned}
& \Delta_{\xi} \tilde{y}(\xi)=-\left\{\tilde{y}^{2}(\xi)+\frac{2}{\varepsilon_{0}} \tilde{y}(\xi)\right\}^{3 / 2}, \\
& \xi_{1}\left(x_{0} \mid z\right)=k \xi_{1}\left(x_{0}\right), \quad \mathcal{M}\left(x_{0} \mid z\right)=k^{3} \mathcal{M}\left(x_{0}\right), \\
& \mathcal{M}\left(x_{0}\right)=\int_{0}^{\xi_{1}\left(x_{0}\right)} \xi^{2}\left\{\tilde{y}^{2}(\xi)+\frac{2}{\varepsilon_{0}} \tilde{y}(\xi)\right\}^{3 / 2} d \xi .
\end{aligned}
$$

The dimensionless mass and radius calculated by formulae (18) are shown in Table 3. As was shown in Tables 2 and 3, the relative error of the determination of the mass in the range $x_{0}>5$ does not exceed $1 \%$. At $x_{0}>10$, it is smaller than $0.3 \%$. The calculation error of the radius increases with $z$. At $z=2$, it is at most $0.4 \%$. At $z=26$, it is smaller than $3 \%$.

\section{Influence of Interactions and the Axial Rotation}

The comparison of Tables 2 and 3 shows that Eq. (12) at $\Omega \neq 0$ can be substantially simplified by neglecting the multiplier $\varphi_{2}(\xi, \theta \mid z)$ and by replacing the term proportional to $\varphi_{1}(\xi, \theta \mid z)$ by $\varphi_{1}\left(x_{0} \mid z\right) \Delta(\xi, \theta) Y(\xi, \theta)$ without accuracy loss. One can introduce the dimensionless radial coordinate $\tilde{\xi}=r / \tilde{\lambda}$, where $\tilde{\lambda}$ is determined from the equation

$\frac{32 \pi^{2} G}{3(h c)^{3}}\left(m_{u} \mu_{e} m_{0} c^{2} \varepsilon_{0} \tilde{\lambda}\right)^{2}=1-\varphi_{1}\left(x_{0} \mid z\right)$.

Then Eq. (12) takes the form

$\Delta(\tilde{\xi}, \theta) \tilde{Y}(\tilde{\xi}, \theta)=\tilde{\Omega}^{2}-\left\{\tilde{Y}^{2}(\tilde{\xi}, \theta)+\frac{2}{\varepsilon_{0}} \tilde{Y}(\tilde{\xi}, \theta)\right\}^{3 / 2}$,

where

$\tilde{\Omega}^{2}=\frac{2 \omega^{2} m_{u} \mu_{e}}{m_{0} c^{2} \varepsilon_{0}} \tilde{\lambda}^{2}=\Omega^{2}\left(1-\varphi_{1}\left(x_{0} \mid z\right)\right)$.

Formally, Eq. (20) coincides with the equilibrium equation of a degenerate dwarf with axial rotation in the standard model written in the dimensionless form. The solution of Eq. (12) is

$Y(\xi, \theta)=Y(\tilde{\xi} k, \theta)=\tilde{Y}(\tilde{\xi}, \theta)$,

where $\tilde{Y}(\tilde{\xi}, \theta)$ is a solution of Eq. (20), and $k=$ $=\left[1-\varphi_{1}\left(x_{0} \mid z\right)\right]^{1 / 2}$. Therefore, we will omit " $\sim$ " over the variable $\tilde{\xi}$, while looking for the solutions of ISSN 2071-0194. Ukr. J. Phys. 2018. Vol. 63, No. 9

\begin{tabular}{|c|c|c|c|c|}
\hline$x_{0}$ & $z=2$ & $z=6$ & $z=12$ & $z=26$ \\
\hline \multicolumn{5}{|c|}{$\mathcal{M}\left(x_{0} \mid z\right)$} \\
\hline 1.0 & 0.698071 & 0.689712 & 0.680305 & 0.663335 \\
\hline 2.0 & 1.22614 & 1.21149 & 1.19504 & 1.16539 \\
\hline 3.0 & 1.49846 & 1.48056 & 1.46048 & 1.42434 \\
\hline 4.0 & 1.64937 & 1.62967 & 1.60756 & 1.5678 \\
\hline 5.0 & 1.74063 & 1.71984 & 1.6965 & 1.65452 \\
\hline 6.0 & 1.79981 & 1.77832 & 1.75418 & 1.71075 \\
\hline 7.0 & 1.84033 & 1.81835 & 1.79366 & 1.74923 \\
\hline 8.0 & 1.86927 & 1.84694 & 1.82186 & 1.77671 \\
\hline 9.0 & 1.89065 & 1.86807 & 1.84269 & 1.79701 \\
\hline 10.0 & 1.9069 & 1.88412 & 1.85852 & 1.81242 \\
\hline 15.0 & 1.94967 & 1.92639 & 1.9002 & 1.853 \\
\hline 20.0 & 1.9667 & 1.9432 & 1.91678 & 1.86913 \\
\hline 25.0 & 1.97512 & 1.95153 & 1.92498 & 1.8771 \\
\hline 30.0 & 1.97988 & 1.95623 & 1.92961 & 1.8816 \\
\hline \multicolumn{5}{|c|}{$\xi_{1}\left(x_{0} \mid z\right)$} \\
\hline 1.0 & 1.03067 & 1.02654 & 1.02185 & 1.01328 \\
\hline 2.0 & 2.05071 & 2.04251 & 2.03323 & 2.01627 \\
\hline 3.0 & 2.76986 & 2.75879 & 2.74626 & 2.72342 \\
\hline 4.0 & 3.29291 & 3.27975 & 3.26485 & 3.23771 \\
\hline 5.0 & 3.69122 & 3.67647 & 3.65977 & 3.62933 \\
\hline 6.0 & 4.00587 & 3.98986 & 3.97173 & 3.93868 \\
\hline 7.0 & 4.26151 & 4.24447 & 4.22518 & 4.19000 \\
\hline 8.0 & 4.47378 & 4.4559 & 4.43563 & 4.39868 \\
\hline 9.0 & 4.65315 & 4.63455 & 4.61347 & 4.57502 \\
\hline 10.0 & 4.80689 & 4.78768 & 4.7659 & 4.72616 \\
\hline 15.0 & 5.33474 & 5.31341 & 5.28922 & 5.24507 \\
\hline 20.0 & 5.64552 & 5.62295 & 5.59735 & 5.55058 \\
\hline 25.0 & 5.85091 & 5.82752 & 5.80097 & 5.75247 \\
\hline 30.0 & 5.99691 & 5.97294 & 5.94573 & 5.896 \\
\hline
\end{tabular}

Table 3. Dependence of $\mathcal{M}\left(\boldsymbol{x}_{0} \mid \boldsymbol{z}\right)$ and $\boldsymbol{\xi}_{1}\left(\boldsymbol{x}_{0} \mid \boldsymbol{z}\right)$ on the relativistic parameter and the nuclear charge

Eq. (20). In the case of massive degenerate dwarf, Eq. (20) has two small parameters $\tilde{\Omega}^{2}$ and $\varepsilon_{0}^{-1}=$ $=\left[\left(1+x_{0}^{2}\right)^{1 / 2}-1\right]^{-1}$. In the limit $\tilde{\Omega} \rightarrow 0, x_{0} \rightarrow \infty$, Eq. (20) transforms to the equation of the polytropic model with index $n=3$. At $\tilde{\Omega} \neq 0$ and $x_{0} \rightarrow \infty$, Eq. (20) describes the equilibrium in the polytropic model (with index $n=3$ ), i.e., one rotating with constant angular velocity $\tilde{\Omega}^{2}$.

The solutions of an equilibrium equation in the standard model (Eq. (15) at $\varphi_{1}(\xi \mid z)=\varphi_{2}(\xi \mid z)=0$ ) for massive dwarfs can be expanded in the small parameter $\varepsilon_{0}^{-1}$ :

$y(\xi)=y_{0}(\xi)+\frac{y_{1}(\xi)}{\varepsilon_{0}}+\frac{y_{2}(\xi)}{\varepsilon_{0}^{2}}+\ldots$ 


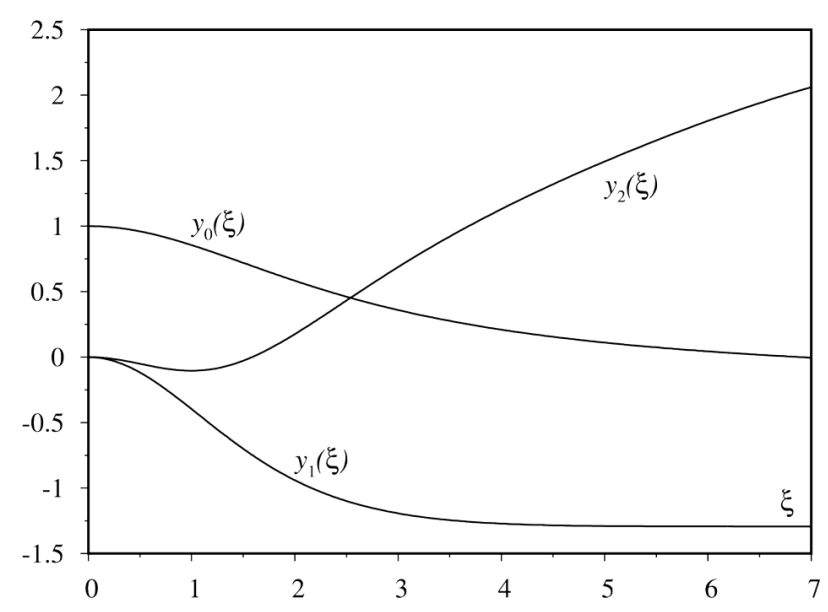

Fig. 2. Numerical solutions of the system of equations (24)

The functions $y_{0}(\xi), y_{i}(\xi)$ are determined from the chain of equations

$$
\begin{aligned}
& \Delta_{\xi} y_{0}(\xi)+y_{0}^{3}(\xi)=0 \\
& \Delta_{\xi} y_{1}(\xi)+3 y_{1}(\xi) y_{0}^{2}(\xi)=-3 y_{0}^{2}(\xi) \\
& \Delta_{\xi} y_{2}(\xi)+3 y_{2}(\xi) y_{0}^{2}(\xi)= \\
& =-\frac{3}{2} y_{0}(\xi)\left\{1+4 y_{1}(\xi)+2 y_{1}^{2}(\xi)\right\} ; \ldots
\end{aligned}
$$

with boundary conditions: $y_{0}(0)=1 ; y_{l}(0)=0$ at $l \geq 1 ; y_{l}^{\prime}(0)=0$ at $l \geq 0$. The functions $y_{l}(\xi)$ obtained numerically are depicted in Fig. 2. They do not have any parameters and represent a universal basis for calculation of the dwarf characteristics in the standard model as functions of the parameters $x_{0}$ and $\mu_{e}$. Herewith, $y_{0}(\xi)$ is a solution of the equilibrium equation in the polytropic model with $n=3$.

Similarly, the solution of Eq. (20) can be represented as the expansion

$Y(\xi, \theta)=Y_{3}(\xi, \theta)+\sum_{l \geq 1} \varepsilon_{0}^{-l}\left(x_{0}\right) F_{l}(\xi, \theta)$,

where $Y_{3}(\xi, \theta)$ is a solution of the equilibrium equation for the polytrope with index $n=3$ in the presence of a rotation, and $F_{l}(\xi, \theta)$ are the unknown functions. Substituting series (25) in Eq. (20) and expanding the expression $\left\{Y^{2}+2 Y / \varepsilon_{0}\right\}^{3 / 2}$ in powers of $\varepsilon_{0}^{-1}$, we obtain the following chain of equations:

$$
\begin{aligned}
& \Delta Y_{3}(\xi, \theta)+Y_{3}^{3}(\xi, \theta)=\tilde{\Omega}^{2}, \\
& \Delta F_{1}(\xi, \theta)+3 F_{1}(\xi, \theta) Y_{3}^{2}(\xi, \theta)=-3 Y_{3}^{2}(\xi, \theta),
\end{aligned}
$$

$\Delta F_{2}(\xi, \theta)+3 F_{2}(\xi, \theta) Y_{3}^{2}(\xi, \theta)=$

$=-\frac{3}{2} Y_{3}(\xi, \theta)\left\{1+4 F_{1}(\xi, \theta)+2 F_{1}^{2}(\xi, \theta)\right\}$,

where $\Delta \equiv \Delta(\xi, \theta)$. The substitutions

$Y_{3}(\xi, \theta)=y_{0}(\xi)+\tilde{\Omega}^{2} f_{0}(\xi, \theta) ;$

$F_{l}(\xi, \theta)=y_{l}(\xi)+\tilde{\Omega}^{2} f_{l}(\xi, \theta)$ by $l \geq 1$

are justified, because the parameter $\tilde{\Omega}^{2}$ is small for massive dwarfs. With these substitutions, we can exclude the parameter $\tilde{\Omega}^{2}$ from system (26) and rewrite it in a universal form:

$\Delta f_{0}(\xi, \theta)+3 f_{0}(\xi, \theta) y_{0}^{2}(\xi)=1 ;$

$\Delta f_{1}(\xi, \theta)+3 f_{1}(\xi, \theta) y_{0}^{2}(\xi)=$

$=-6 y_{0}(\xi) f_{0}(\xi, \theta)\left[1+y_{1}(\xi)\right]$

$\Delta f_{2}(\xi, \theta)+3 f_{2}(\xi, \theta) y_{0}^{2}(\xi)=$

$=-6 f_{1}(\xi, \theta)\left[1+y_{1}(\xi)\right]-6 f_{0}(\xi, \theta) y_{0}(\xi) y_{2}(\xi) ; \ldots$.

We have used another substitution, namely

$f_{0}(\xi, \theta)=\psi_{0,0}(\xi)+A P_{2}(\cos \theta) \psi_{0,2}(\xi)$,

$f_{l}(\xi, \theta)=\psi_{l, 0}(\xi)+P_{2}(\cos \theta) \psi_{l, 2}(\xi)$,

where $P_{2}(\cos \theta)$ is the Legendre polynomial of the second order, $l \geq 1$. Substitutions (29) allow us to separate the variables in Eqs. (28) and to get a chain of equations for the functions $\psi_{l, 0}(\xi), \psi_{l, 2}(\xi)$ at $l \geq 0$. Only the equation for $\psi_{0,2}(\xi)$ is linear and homogeneous:

$\Delta_{\xi} \psi_{0,2}(\xi)=\psi_{0,2}(\xi)\left\{3 y_{0}^{2}(\xi)+\frac{6}{\xi^{2}}\right\}$.

All other functions are solutions of the linear inhomogeneous equations:

$\Delta_{\xi} \psi_{0,0}(\xi)+3 y_{0}^{2}(\xi) \psi_{0,0}(\xi)=1 ;$

$\Delta_{\xi} \psi_{1,0}(\xi)+3 y_{0}^{2}(\xi) \psi_{1,0}(\xi)=$

$=-6\left[1+y_{1}(\xi)\right] y_{0}(\xi) \psi_{0,0}(\xi)$;

$\Delta_{\xi} \psi_{2,0}(\xi)+3 y_{0}^{2}(\xi) \psi_{2,0}(\xi)=-6 \psi_{1,0}(\xi)\left[1+y_{1}(\xi)\right]-$

$-6 y_{0}(\xi) y_{2}(\xi) \psi_{0,0}(\xi)$;

$\Delta_{\xi} \psi_{1,2}(\xi)-\frac{6}{\xi^{2}} \psi_{1,2}(\xi)+3 y_{0}^{2} \psi_{1,2}(\xi)=$

$=-6 A \psi_{0,2}(\xi)\left[1+y_{1}(\xi)\right]$

$\Delta_{\xi} \psi_{2,2}(\xi)-\frac{6}{\xi^{2}} \psi_{2,2}(\xi)+3 y_{0}^{2} \psi_{2,2}(\xi)=$

$-6\left[1+y_{1}(\xi)\right] \psi_{0,2}(\xi)-6 A y_{0}(\xi) y_{2}(\xi) \psi_{0,2}(\xi) ;$

ISSN 2071-0194. Ukr. J. Phys. 2018. Vol. 63, No. 9 
According to (14), all functions $\psi_{l, 0}(\xi), \psi_{l, 2}(\xi)$ at $l \geq$ $\geq 0$ satisfy the boundary conditions $\psi_{l, 0}(0)=\psi_{l, 2}(0)=$ $=0, \frac{\partial}{\partial \xi} \psi_{l, 0}(\xi)=\frac{\partial}{\partial \xi} \psi_{l, 2}(\xi)=0$ at $\xi=0$.

In work [14] devoted to the polytropic stars with axial rotation, the functions $\psi_{0,0}(\xi)$ and $\psi_{0,2}(\xi)$ were calculated by the numerical integration, and the constant of integration $A=-0.72325$ was determined. Using the same method, we have found the solutions of the equation for $\psi_{l, 0}(\xi), \psi_{l, 2}(\xi)$ at $l=1$ and 2 . The results of calculations for the universal functions $y_{l}(\xi), \psi_{l, 0}(\xi)$ and $\psi_{l, 2}(\xi)$ at $l \geq 0$ have been presented in the form of the Pade-approximants given in Appendix 2. The functions $y_{l}(\xi), \psi_{l, 0}(\xi)$ and $\psi_{l, 2}(\xi)$ do not depend on any parameters and form a universal system, which makes it possible to present all the characteristics of massive dwarfs with axial rotation in the form of an expansion in powers of the small parameter $\varepsilon_{0}^{-1}\left(x_{0}\right)$.

According to Eq. (25), the solution of (20) reads

$\tilde{Y}(\xi, \theta)=y\left(\xi \mid x_{0}\right)+$

$+\tilde{\Omega}^{2}\left\{\Psi_{0}\left(\xi \mid x_{0}\right)+P_{2}(\cos \theta) \Psi_{2}\left(\xi \mid x_{0}\right)\right\}$,

where $y\left(\xi \mid x_{0}\right)$ is a solution of the equilibrium equation for a degenerate dwarf in the standard model,

$$
\begin{aligned}
& \Psi_{0}\left(\xi \mid x_{0}\right)=\psi_{0,0}(\xi)+\sum_{l \geq 1} \psi_{l, 0}(\xi) \varepsilon_{0}^{-l}\left(x_{0}\right) \\
& \Psi_{2}\left(\xi \mid x_{0}\right)=A \psi_{0,2}(\xi)+\sum_{l \geq 1} \psi_{l, 2}(\xi) \varepsilon_{0}^{-l}\left(x_{0}\right) .
\end{aligned}
$$

The conditions

$\tilde{Y}\left(\xi, \frac{\pi}{2}\right)=0, \quad \frac{\partial}{\partial \xi} \tilde{Y}\left(\xi, \frac{\pi}{2}\right)=0$

determine the maximal value of the angular velocity $\tilde{\Omega}_{\max }\left(x_{0}\right)$ and corresponding maximal value of the dimensionless equatorial radius $\xi_{e}^{\max }\left(x_{0}\right)$. At $\tilde{\Omega}>$ $>\tilde{\Omega}_{\max }$, the stability of a star is disturbed in a vicinity of the equator, and the function $\tilde{Y}(\xi, \theta)$ becomes a non-monotonous function of $\xi$. The root of the equation $\tilde{Y}(\xi, \theta)=0$ at $\tilde{\Omega}<\tilde{\Omega}_{\max }$ determines the shape of a star,

$\xi_{1}(\theta) \equiv \xi_{1}\left(\theta \mid x_{0}, \tilde{\Omega}\right)$.

The dependence of function (32) on the variables $(\xi, \theta)$ is illustrated in Fig. 3 for $\theta=0$ and $\theta=\pi / 2$ in the case $\tilde{\Omega}_{\max }^{2}\left(x_{0}\right)$ at $x_{0}=10$. The solution of the standard model is given for comparison. One can

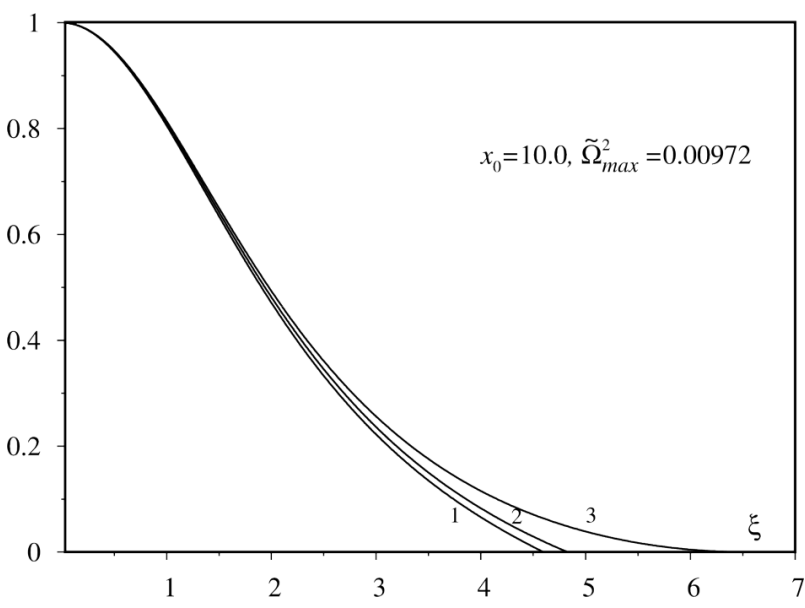

Fig. 3. Dependence of function (32) at a fixed value of $x_{0}$ and $\tilde{\Omega}_{\max }^{2}$ (curve 1 corresponds to the angle $\theta=0$, curve 2 shows solutions of the equilibrium equation in the standard model, curve 3 presents the angle $\theta=\pi / 2$ )

Table 4. Dependence of the maximal value of the parameter $\tilde{\Omega}^{2}$ corresponding to the dimensionless equatorial and polar radii, as well as the dimensionless radius of the dwarf without rotation, on the parameter $x_{0}$

\begin{tabular}{|r|l|l|c|c|}
\hline$x_{0}$ & $\tilde{\Omega}_{\max }^{2}$ & $\xi_{1}\left(x_{0}\right)$ & $\xi_{e}\left(x_{0} \mid \tilde{\Omega}_{\max }^{2}\right)$ & $\xi_{p}\left(x_{0} \mid \tilde{\Omega}_{\max }^{2}\right)$ \\
\hline 6.0 & 0.0164 & 4.023 & 5.401 & 3.801 \\
8.0 & 0.0119 & 4.493 & 6.001 & 4.271 \\
10.0 & 0.00972 & 4.828 & 6.461 & 4.591 \\
15.0 & 0.00733 & 5.358 & 7.231 & 5.111 \\
20.0 & 0.00632 & 5.670 & 7.651 & 5.421 \\
25.0 & 0.00579 & 5.887 & 8.021 & 5.631 \\
\hline
\end{tabular}

find the dependence of $\tilde{\Omega}_{\max }^{2}$, as well as the equatorial $\xi_{e}\left(x_{0} \mid \tilde{\Omega}_{\max }^{2}\right)$ and polar $\xi_{p}\left(x_{0} \mid \tilde{\Omega}_{\max }^{2}\right)$ radii, on the relativistic parameter $x_{0}$ (see Table 4$)$.

The approximate solution of Eq. (12) has been obtained by replacing $\xi \rightarrow k \xi$ in expression (32), where $k=\left[1-\varphi_{1}\left(x_{0} \mid z\right)\right]^{1 / 2}$. Therefore, the mass of a degenerate dwarf in a model with interactions is determined by the expression

$M\left(x_{0}, \mu_{e}, z, \omega\right)=\frac{M_{0}}{\mu_{e}^{2}}\left(1-\varphi_{1}\left(x_{0} \mid z\right)\right)^{3 / 2} \mathcal{M}\left(x_{0} \mid \tilde{\Omega}\right)$,

where

$$
\begin{aligned}
& \mathcal{M}\left(x_{0} \mid \tilde{\Omega}\right)=\int_{0}^{1} d t \int_{0}^{\xi_{1}\left(\theta \mid x_{0}, \tilde{\Omega}\right)} \xi^{2} d \xi \times \\
& \times\left\{\tilde{Y}^{2}(\xi, \theta)+\frac{2}{\varepsilon_{0}} \tilde{Y}(\xi, \theta)\right\}^{3 / 2},
\end{aligned}
$$




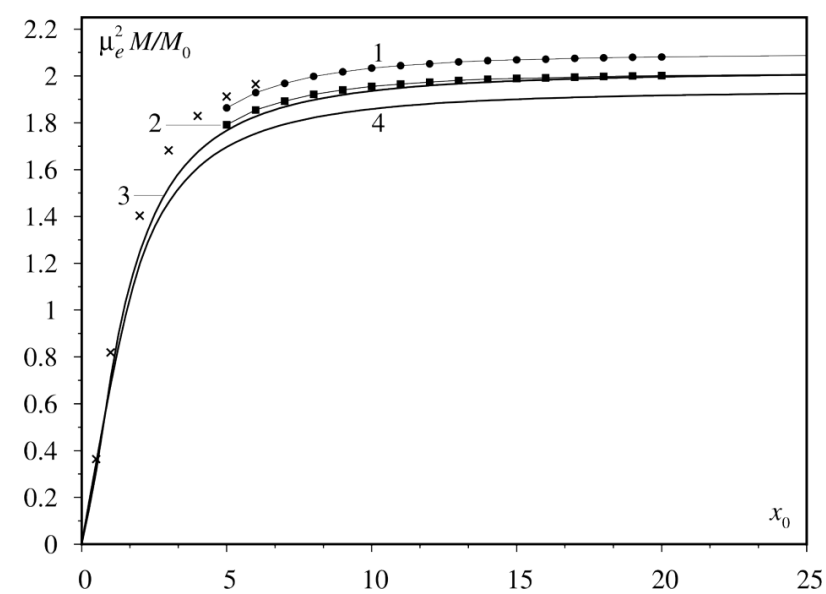

Fig. 4. Dependence of dwarf's mass on the relativistic parameter in different approximations (see the text)

and the polar and the equatorial radii are

$$
\begin{aligned}
& R_{p}\left(x_{0}, \mu_{e}, z, \omega\right)=\frac{R_{0}}{\mu_{e} \varepsilon_{0}} \xi_{p}\left(x_{0}, z, \tilde{\Omega}\right), \\
& R_{e}\left(x_{0}, \mu_{e}, z, \omega\right)=\frac{R_{0}}{\mu_{e} \varepsilon_{0}} \xi_{e}\left(x_{0}, z, \tilde{\Omega}\right), \\
& \xi_{p}\left(x_{0}, z, \tilde{\Omega}\right)=\left[1-\varphi_{1}\left(x_{0} \mid z\right)\right]^{1 / 2} \xi_{1}\left(0 \mid x_{0}, \tilde{\Omega}\right), \\
& \xi_{e}\left(x_{0}, z, \tilde{\Omega}\right)=\left[1-\varphi_{1}\left(x_{0} \mid z\right)\right]^{1 / 2} \xi_{1}\left(\pi / 2 \mid x_{0}, \tilde{\Omega}\right) .
\end{aligned}
$$

The dimensionless radius $\xi_{e, p}\left(x_{0}, z, \tilde{\Omega}\right)$ calculated at $\tilde{\Omega}=\tilde{\Omega}_{\max }\left(x_{0}\right)$ is given in Table 5 .

The influence of interactions and the axial rotation on the dwarf mass as a function of the parameter $x_{0}$ is shown in Fig. 4. Curve 1 corresponds to the dimensionless mass of a dwarf rotating with maximal angular velocity $\tilde{\Omega}_{\max }\left(x_{0}\right)$ without interactions $\left(\mathcal{M}\left(x_{0} \mid \tilde{\Omega}_{\max }\right)\right)$. The crosses correspond to the

Table 5. Dependence of the dimensionless equatorial and polar radii on the relativistic parameter $x_{0}$ and the charge of nucleus $z$

\begin{tabular}{|r|c|c|c|c|c|c|}
\hline \multirow{2}{*}{$x_{0}$} & \multicolumn{3}{|c|}{$\xi_{e}\left(x_{0}, z, \tilde{\Omega}\right)$} & \multicolumn{3}{c|}{$\xi_{p}\left(x_{0}, z, \tilde{\Omega}\right)$} \\
\cline { 2 - 7 } & $z=2$ & $z=12$ & $z=26$ & $z=2$ & $z=12$ & $z=26$ \\
\hline 6.0 & 5.377 & 5.331 & 5.287 & 3.784 & 3.752 & 3.721 \\
8.0 & 5.974 & 5.923 & 5.874 & 4.252 & 4.216 & 4.181 \\
10.0 & 6.432 & 6.377 & 6.324 & 4.570 & 4.531 & 4.494 \\
15.0 & 7.199 & 7.137 & 7.078 & 5.088 & 5.045 & 5.003 \\
20.0 & 7.617 & 7.552 & 7.489 & 5.397 & 5.351 & 5.306 \\
25.0 & 7.985 & 7.917 & 7.851 & 5.606 & 5.558 & 5.511 \\
\hline
\end{tabular}

mass value, which was calculated in work [7] for several values of the relativistic parameter in the region $0.5 \leq x_{0} \leq 6.24$. Curve 2 corresponds to formula (36) and involves both factors, the interactions and the rotation with $\tilde{\Omega}_{\max }\left(x_{0}\right)$ at $z=12$. Curve 3 corresponds to the standard model and considers neither interactions nor rotation $\left(\mathcal{M}\left(x_{0}\right)\right)$. Curve 4 is constructed according to formula (18) at $z=12$ and involves the influence of interactions in the dwarf model without rotation. As can be seen from the figure, the maximal mass of a dwarf at the maximal rotation velocity exceeds the mass in the standard model without rotation and interactions approximately by a factor of $5.4 \%$ at $x_{0}=10 ; 4.4 \%$ at $x_{0}=20 ;$ and $4.1 \%$ at $x_{0}=30$.

The dependences of dwarf's mass on the $x_{0}$ in Chandrasekhar's model $\mathcal{M}\left(x_{0}\right)$ [the model without rotation, but accounting the interactions $[1-$ $\left.\left.-\varphi_{1}\left(x_{0}, z\right)\right]^{3 / 2} \mathcal{M}\left(x_{0}\right)\right]$, the maximal mass in the model with rotation (but without the interactions) $\mathcal{M}\left(x_{0}, \tilde{\Omega}_{\max }\right)$, and the mass in the model with rotation and interactions $\left[1-\varphi_{1}\left(x_{0}, z\right)\right]^{3 / 2} \mathcal{M}\left(x_{0}, \tilde{\Omega}_{\max }\right)$ are given in Table 6.

\section{Stability of Degenerate Dwarfs}

The maximal mass of dwarfs is related to the problem of their stability. There are two main causes for the instability of such star at high densities. The first of them is the neutronization process, which leads to a decrease of the electrons concentration. The threshold value of the relativistic parameter at the center of the star

$x_{0}=a_{0} \alpha_{0}\left(3 \pi^{2} \frac{\rho_{0}}{m_{u} \mu_{e}}\right)^{1 / 3}$,

where $a_{0}$ is the Bohr radius, $\alpha_{0}$ is the fine structure constant, and $\rho_{0}$ is the threshold density of neutronization reaction. In Table 7 , we give the value of $\rho_{0}$ for several chemical elements (in $\mathrm{g} / \mathrm{cm}^{3}$ ) taken from work [15] and the calculated threshold value of the relativistic parameter $x_{0}$,

$x_{0}=\left(\frac{2}{\mu_{e}}\right)^{1 / 3} 0.7976 \times 10^{-2} \rho_{0}^{1 / 3}$.

The influence of the neutronization was considered in [16].

The second cause for the instability is the effects of general theory of relativity (GTR). Restrictions

ISSN 2071-0194. Ukr. J. Phys. 2018. Vol. 63, No. 9 
on the degenerate dwarf mass involving the effects of GTR were first considered in work [6] in the approximation of Chandrasehkar's model (without interactions and rotation). For the consideration of the interactions, we use the Oppenheimer-Volkoff equations

$$
\begin{aligned}
& \frac{d P}{d r}=-G \rho(r) \frac{M(r)}{r^{2}}\left(1+\frac{P(r)}{\rho(r) c^{2}}\right) \times \\
& \times\left(1+4 \pi r^{3} \frac{P(r)}{M(r) c^{2}}\right)\left(1-2 G \frac{M(r)}{r c^{2}}\right)^{-1}, \\
& \frac{d M}{d r}=4 \pi r^{2} \rho(r),
\end{aligned}
$$

in which $M(r)$ is the mass in a sphere of radius $r$. Let us substitute the expression for the pressure (6) in the left-hand side of the first equation and expand the right-hand side in a series in $c^{-2}$, by retaining only linear terms and putting $f(x(r) \mid z)=0$ in them. In the dimensionless variables (8), Eq. (41) is reduced to the differential equation

$$
\begin{aligned}
& \frac{1}{\xi^{2}} \frac{d}{d \xi}\left\{\xi^{2} \frac{d y}{d \xi}-\xi^{2} \varphi(\xi \mid z) \frac{d}{d \xi}\left[y^{2}+\frac{2}{\varepsilon_{0}} y\right]^{1 / 2}\right\}= \\
& =-\left\{y^{2}+\frac{2}{\varepsilon_{0}} y\right\}^{3 / 2}\left\{1+\gamma\left(x_{0}\right) g(\xi)\right\}- \\
& -\frac{\gamma\left(x_{0}\right)}{\xi^{2}} \mathcal{M}(\xi) \frac{d g(\xi)}{d \xi} .
\end{aligned}
$$

Here, we used the notations

$$
\begin{aligned}
& \varphi(\xi \mid z)=(2 x)^{-3} \frac{d}{d x} f(x \mid z) \text { by } x=\varepsilon_{0}\left(y^{2}+\frac{2}{\varepsilon_{0}} y\right)^{1 / 2} \\
& g(\xi)=\frac{1}{4}\left(y^{2}+\frac{2}{\varepsilon_{0}} y\right)^{1 / 2}+ \\
& +\frac{2}{\xi} \mathcal{M}(\xi)+\frac{\xi^{3}}{4 \mathcal{M}(\xi)}\left(y^{2}+\frac{2}{\varepsilon_{0}} y\right)^{2} ; \\
& \mathcal{M}(\xi)=\int_{0}^{\xi}\left(\xi^{\prime}\right)^{2}\left\{y^{2}\left(\xi^{\prime}\right)+\frac{2}{\varepsilon_{0}} y\left(\xi^{\prime}\right)\right\}^{3 / 2} d \xi^{\prime} .
\end{aligned}
$$

In the nonlinear integral-differential equation (42), two independent parameters $x_{0}$ and $z$ appear, as well as the dimensionless parameter

$\gamma\left(x_{0}\right)=\varepsilon_{0}\left(x_{0}\right) \frac{m_{0}}{m_{u} \mu_{e}} \sim \frac{x_{0}}{4} \times 10^{-3}$.
Substituting $\varphi(\xi \mid z)$ to $\varphi\left(x_{0} \mid z\right)$ and introducing the new variable $\tilde{\xi}=\xi / k\left(\right.$ where $\left.k=\left[1-\varphi\left(x_{0} \mid z\right)\right]^{1 / 2}\right)$,

Table 6. Dependence of the dimensionless dwarf mass on the parameter $x_{0}$ in different approximations: $\mathcal{M}\left(x_{0}\right),\left(1-\varphi_{1}\left(x_{0}, z\right)\right)^{3 / 2} \mathcal{M}\left(x_{0}\right)$, $\mathcal{M}\left(x_{0}, \tilde{\Omega}_{\max }\right),\left(1-\varphi_{1}\left(x_{0}, z\right)\right)^{3 / 2} \mathcal{M}\left(x_{0}, \tilde{\Omega}_{\max }\right)$ (see text)

\begin{tabular}{|r|c|l|l|l|}
\hline & \multirow{2}{*}{$x_{0}$} & $\mathcal{M}\left(x_{0}\right)$ & \multicolumn{3}{|c|}{$\left(1-\varphi_{1}\left(x_{0}, z\right)\right)^{3 / 2} \mathcal{M}\left(x_{0}\right)$} \\
\cline { 3 - 5 } & & $z=2$ & $z=12$ & $z=26$ \\
\hline 5.0 & 1.76395 & 1.740419 & 1.696298 & 1.654323 \\
6.0 & 1.82404 & 1.799697 & 1.754065 & 1.710638 \\
7.0 & 1.86521 & 1.840310 & 1.7936401 & 1.7492106 \\
8.0 & 1.89462 & 1.869321 & 1.821909 & 1.776757 \\
9.0 & 1.91634 & 1.890746 & 1.842785 & 1.797095 \\
10.0 & 1.93284 & 1.907022 & 1.858643 & 1.812542 \\
15.0 & 1.97619 & 1.949781 & 1.900299 & 1.853099 \\
20.0 & 1.99337 & 1.966725 & 1.916804 & 1.869154 \\
25.0 & 2.00186 & 1.975098 & 1.924957 & 1.877079 \\
30.0 & 2.00665 & 1.979821 & 1.929557 & 1.881546
\end{tabular}

\begin{tabular}{|r|c|c|c|c|}
\hline \multirow{2}{*}{$x_{0}$} & $\mathcal{M}\left(x_{0}, \tilde{\Omega}_{\max }\right)$ & \multicolumn{3}{|c|}{$\left(1-\varphi_{1}\left(x_{0}, z\right)\right)^{3 / 2} \mathcal{M}\left(x_{0}, \tilde{\Omega}_{\max }\right)$} \\
\cline { 3 - 5 } & & $z=2$ & $z=12$ & $z=26$ \\
\hline 5.0 & 1.863482 & 1.838623 & 1.792012 & 1.747669 \\
6.0 & 1.928908 & 1.903166 & 1.854910 & 1.808987 \\
7.0 & 1.968121 & 1.941846 & 1.892602 & 1.845721 \\
8.0 & 1.998099 & 1.971418 & 1.921417 & 1.873799 \\
9.0 & 2.017770 & 1.990821 & 1.940322 & 1.892214 \\
10.0 & 2.033656 & 2.006491 & 1.955589 & 1.907083 \\
15.0 & 2.068962 & 2.041313 & 1.989509 & 1.940093 \\
20.0 & 2.081174 & 2.053355 & 2.001235 & 1.951486 \\
25.0 & 2.087382 & 2.059477 & 2.007194 & 1.957270 \\
30.0 & 2.088186 & 2.060267 & 2.007960 & 1.957998 \\
\hline
\end{tabular}

Table 7. Threshold of of the neutronization process for several chemical elements

\begin{tabular}{|c|c|c|}
\hline Nucleus & $\rho_{0}$ & $x_{0}$ \\
\hline${ }_{2}^{4} \mathrm{He}$ & $1.37 \times 10^{11}$ & 41.117 \\
${ }_{6}^{12} \mathrm{C}$ & $3.90 \times 10^{10}$ & 27.048 \\
${ }_{8}^{16} \mathrm{O}$ & $1.90 \times 10^{10}$ & 21.283 \\
${ }_{10}^{20} \mathrm{Ne}$ & $6.21 \times 10^{9}$ & 14.661 \\
${ }_{12}^{24} \mathrm{Mg}$ & $1.97 \times 10^{9}$ & 9.999 \\
${ }_{26}^{56} \mathrm{Fe}$ & $1.14 \times 10^{9}$ & 8.332 \\
\hline
\end{tabular}




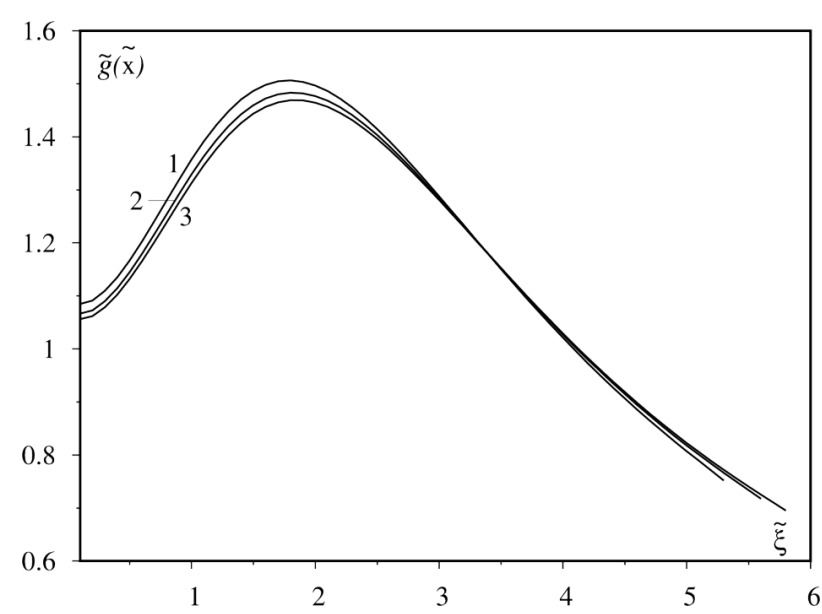

Fig. 5. Dependence of the function $\tilde{g}(\tilde{\xi})$ on the variable $\tilde{\xi}$ at different values of the relativistic parameter $x_{0}$ (curve 1 $\left.x_{0}=15 ; 2-x_{0}=20 ; 3-x_{0}=25\right)$

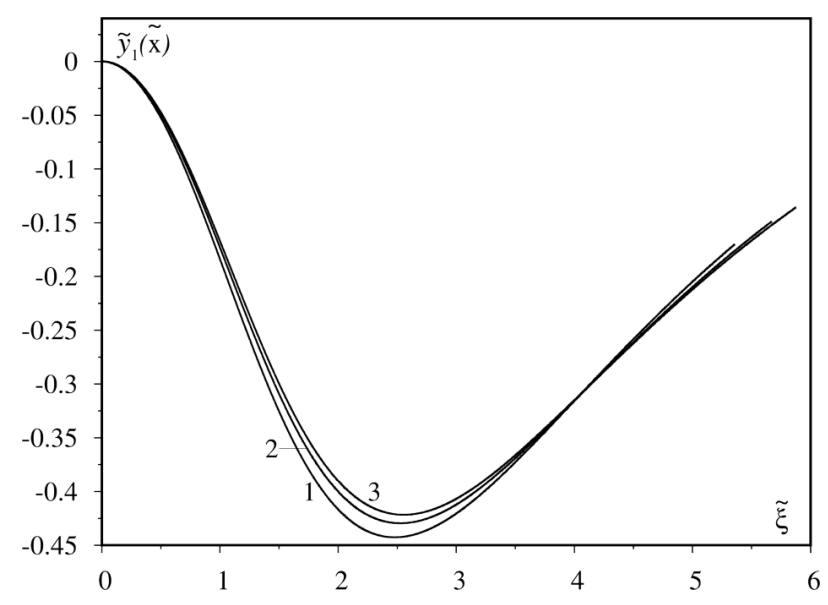

Fig. 6. Solutions of Eq. (50) at different values of the relativistic parameter $x_{0}$ (curve $1-x_{0}=15 ; 2-x_{0}=20 ; 3-$ $\left.x_{0}=25\right)$

we transform (42) to the form

$$
\begin{aligned}
& \frac{1}{\tilde{\xi}^{2}} \frac{d}{d \tilde{\xi}}\left(\tilde{\xi}^{2} \frac{d}{d \tilde{\xi}} \tilde{y}\right)=-\left\{\tilde{y}^{2}+\frac{2}{\varepsilon_{0}} \tilde{y}\right\}^{3 / 2} \times \\
& \times\left\{1+\gamma\left(x_{0}\right) \tilde{g}(\tilde{\xi})\right\}-\frac{\gamma\left(x_{0}\right)}{\tilde{\xi}^{2}} \tilde{\mathcal{M}}(\tilde{\xi}) \frac{d \tilde{g}(\tilde{\xi})}{d \tilde{\xi}}
\end{aligned}
$$

where

$\tilde{\mathcal{M}}(\tilde{\xi})=\int_{0}^{k \tilde{\xi}}\left(\xi^{\prime}\right)^{2}\left\{\tilde{y}^{2}+\frac{2}{\varepsilon_{0}} \tilde{y}\right\}^{3 / 2} d \xi^{\prime}$, and $\tilde{g}(\tilde{\xi})$ is related to $\tilde{y}(\tilde{\xi})$ by the expression

$$
\begin{aligned}
& \tilde{g}(\tilde{\xi})=\frac{1}{4}\left(\tilde{y}^{2}+\frac{2}{\varepsilon_{0}} \tilde{y}\right)^{1 / 2}+\frac{2 k^{2}}{\tilde{\xi}} \tilde{\mathcal{M}}(\tilde{\xi})+ \\
& +\frac{1}{4 \tilde{\mathcal{M}}(\tilde{\xi})}\left(\tilde{y}^{2}+\frac{2}{\varepsilon_{0}} \tilde{y}\right)^{2} .
\end{aligned}
$$

The terms in Eq. (45), which are proportional to the parameter $\gamma\left(x_{0}\right)$, play the role of small corrections. So, to simplify the finding of the solution, we calculate them, by basing on $\tilde{y}_{0}(\tilde{\xi})$, which satisfies the zero-approximation equation

$$
\frac{1}{\tilde{\xi}^{2}} \frac{d}{d \tilde{\xi}}\left(\tilde{\xi}^{2} \frac{d}{d \tilde{\xi}} \tilde{y}_{0}\right)=-\left\{\tilde{y}_{0}^{2}+\frac{2}{\varepsilon_{0}} \tilde{y}_{0}\right\}^{3 / 2} .
$$

In this approximation, the functions $\tilde{g}(\tilde{\xi})$ and $\frac{d}{d \tilde{\xi}} \tilde{g}(\tilde{\xi})$ are simply the given functions of the variable $\tilde{\xi}$ and the parameter $x_{0}$. The function $\tilde{g}(\tilde{\xi})$ is depicted in Fig. 5.

With the help of the substitution

$\tilde{y}(\tilde{\xi})=\tilde{y}_{0}(\tilde{\xi})+\gamma\left(x_{0}\right) \tilde{y}_{1}(\tilde{\xi})$,

we get the equation for $\tilde{y}_{1}(\tilde{\xi})$ :

$$
\begin{aligned}
& \frac{1}{\tilde{\xi}^{2}} \frac{d}{d \tilde{\xi}}\left(\tilde{\xi}^{2} \frac{d}{d \tilde{\xi}} \tilde{y}_{1}(\tilde{\xi})\right)+3 \tilde{y}_{1}(\tilde{\xi})\left[\tilde{y}_{0}(\tilde{\xi})+\frac{1}{\varepsilon_{0}}\right] \times \\
& \times\left[\tilde{y}_{0}^{2}(\tilde{\xi})+\frac{2}{\varepsilon_{0}} \tilde{y}_{0}(\tilde{\xi})\right]^{1 / 2}= \\
& =-\tilde{g}(\tilde{\xi})\left\{\tilde{y}_{0}^{2}(\tilde{\xi})+\frac{2}{\varepsilon_{0}} \tilde{y}_{0}(\tilde{\xi})\right\}^{3 / 2}-\frac{\tilde{\mathcal{M}}(\tilde{\xi})}{\tilde{\xi}^{2}} \frac{d \tilde{g}(\tilde{\xi})}{d \tilde{\xi}} .
\end{aligned}
$$

The boundary conditions corresponding to this equation are

$\tilde{y}_{1}(0)=0, \quad \frac{d}{d \tilde{\xi}} \tilde{y}_{1}(\tilde{\xi})=0$ by $\tilde{\xi}=0$,

and the asymptotics

$\tilde{y}_{1}(\tilde{\xi})=-\frac{\tilde{\xi}^{2}}{24}\left\{1+\frac{2}{\varepsilon_{0}}\right\}^{2}\left\{1+3\left(1+\frac{2}{\varepsilon_{0}}\right)^{3 / 2}\right\}+\ldots$

at $\tilde{\xi} \ll 1$. Solutions of Eq. (50) obtained by the numerical integration are depicted in Fig. 6.

In the used approximation, the mass of a star

$M\left(x_{0} \mid z\right)=\frac{M_{0}}{\mu_{e}^{2}}\left\{1-\varphi\left(x_{0} \mid z\right)\right\}^{3 / 2} \times$
$\times\left\{\mathcal{M}\left(x_{0}\right)-\gamma\left(x_{0}\right) \mathcal{M}_{1}\left(x_{0}\right)\right\}$,

ISSN 2071-0194. Ukr. J. Phys. 2018. Vol. 63, No. 9 
where $\mathcal{M}\left(x_{0}\right)$ corresponds to the standard model,

$$
\begin{aligned}
& \mathcal{M}_{1}\left(x_{0}\right)=3 \int_{0}^{\xi_{1}\left(x_{0}\right)} \tilde{\xi}^{2} \tilde{y}_{1}(\tilde{\xi})\left[\tilde{y}_{0}(\tilde{\xi})+\frac{1}{\varepsilon_{0}}\right] \times \\
& \times\left[\tilde{y}_{0}^{2}(\tilde{\xi})+\frac{2}{\varepsilon_{0}} \tilde{y}_{0}(\tilde{\xi})\right]^{1 / 2} d \tilde{\xi} .
\end{aligned}
$$

Ratios (53) and (54) determine the mass of a dwarf with regard for the interactions and the effects of GTR, but without rotation. As is shown in Table 6, under the influence of the axial rotation, the mass of a dwarf increases by the magnitude

$$
\begin{aligned}
& \Delta \mathcal{M}\left(x_{0}, z, \tilde{\Omega}\right)=\left\{1-\varphi\left(x_{0} \mid z\right)\right\}^{3 / 2} \times \\
& \times\left\{\mathcal{M}\left(x_{0}, \tilde{\Omega}\right)-\mathcal{M}\left(x_{0}\right)\right\} .
\end{aligned}
$$

Now, the dimensionless mass of a degenerate dwarf in the model with the rotation, interactions, and the GTR effects can be written in the form

$$
\begin{aligned}
& \mathcal{M}^{\mathrm{GTR}}\left(x_{0}, z, \tilde{\Omega}\right)=\mathcal{M}\left(x_{0}, z\right)+\Delta \mathcal{M}\left(x_{0}, z, \tilde{\Omega}\right)= \\
& =\left\{1-\varphi\left(x_{0} \mid z\right)\right\}^{3 / 2}\left\{\mathcal{M}\left(x_{0}, \tilde{\Omega}\right)-\gamma\left(x_{0}\right) \mathcal{M}_{1}\left(x_{0}\right)\right\} .
\end{aligned}
$$

The maximal value of the magnitude corresponds to $\tilde{\Omega}=\tilde{\Omega}_{\max }\left(x_{0}\right)$, and the minimal one to $\tilde{\Omega}=0$. Because the parameter $\gamma\left(x_{0}\right)$ is proportional to $\varepsilon_{0}\left(x_{0}\right)$, the quantity $\mathcal{M}^{\mathrm{GTR}}\left(x_{0}, z, \tilde{\Omega}\right)$ as a function of $x_{0}$ has the maximum at some point $x_{0}^{*}$, which is approximately determined by the equation

$\frac{m_{0}}{m_{u} \mu_{e}} \mathcal{M}_{1}\left(x_{0}^{*}\right) \simeq \frac{d}{d x_{0}^{*}} \mathcal{M}\left(x_{0}^{*}, \Omega\right)$.

We have that $x_{0}^{*}$ is in the region of large values of the relativistic parameter, where the derivative $\frac{d}{d x_{0}} \mathcal{M}\left(x_{0}, \tilde{\Omega}\right)$ is about $10^{-3}$. As can be seen from Table 6 , this derivative decreases with increasing $\tilde{\Omega}$, so the increase of the angular velocity leads to a decrease of $x_{0}^{*}$. Furthermore, $x_{0}^{*}$ almost does not depend on the nuclear charge $z$, in contrast to the maximal value of the mass $\mathcal{M}^{\text {GTR }}\left(x_{0}^{*}, z, \tilde{\Omega}\right)$. In Table 8 , we give the masses with the interactions, $\mathcal{M}^{\mathrm{GTR}}\left(x_{0}^{*}, z, 0\right)$, and with the interactions and rotation, $\mathcal{M}^{\mathrm{GTR}}\left(x_{0}^{*}, z, \tilde{\Omega}\right)$ (SM corresponds to the model without interactions and rotation). As follows from the numerical calcula-

\begin{tabular}{|c|c|c|c|c|}
\hline$x_{0}^{(2)}$ & \multicolumn{4}{|c|}{$\mathcal{M}^{\mathrm{GTR}}\left(x_{0}, z, 0\right)$} \\
\hline 26.2 & $\begin{array}{c}\text { SM } \\
1.97531\end{array}$ & $\begin{array}{c}z=2 \\
1.94889\end{array}$ & $\begin{array}{c}z=12 \\
1.89942\end{array}$ & $\begin{array}{c}z=26 \\
1.85217\end{array}$ \\
\hline$x_{0}^{(1)}$ & \multicolumn{4}{|c|}{$\mathcal{M}^{\mathrm{GTR}}\left(x_{0}, z, \tilde{\Omega}\right)$} \\
\hline 23.2 & $\begin{array}{c}\text { SM } \\
2.06117\end{array}$ & $\begin{array}{c}z=2 \\
2.03362\end{array}$ & $\begin{array}{c}z=12 \\
1.98199\end{array}$ & $\begin{array}{c}z=26 \\
1.93270\end{array}$ \\
\hline
\end{tabular}
tion, $x_{0}^{(1)} \leq x_{0}^{*} \leq x_{0}^{(2)}$ at the change of the angular velocity from $\tilde{\Omega}=\tilde{\Omega}_{\max }$ to $\tilde{\Omega}=0$. The maximal mass
Table 8. Critical parameters

of the degenerate dwarfs in the difference models

of a degenerate dwarf at the fixed value of $z$ varies within $\mathcal{M}^{\mathrm{GTR}}\left(x_{0}^{(2)}, z, 0\right)$ to $\mathcal{M}^{\mathrm{GTR}}\left(x_{0}^{(1)}, z, \tilde{\Omega}\right)$, where $x_{0}^{(1)}=23.2\left(\right.$ at $\left.\tilde{\Omega}=\tilde{\Omega}_{\max }\right) ; x_{0}^{(2)}=26.2($ at $\tilde{\Omega}=0)$. As shown in Table 8 , the change area $x_{0}^{*}$ is small, and $x_{0}^{(1)}$ is close to the threshold values of the parameter $x_{0}$, corresponding to the processes of neutronization (see Table 7).

\section{Conclusions}

We have shown that the influence of the competing factors significantly affects the characteristics and internal structure of degenerate dwarfs. The interactions cause a decrease of the mass of degenerate dwarfs. The axial rotation can partially (depending on the $\tilde{\Omega}$ ) compensate the influence of the Coulomb interactions at $z \lesssim 15$. In this case, the dwarf mass can exceed Chandrasekhar's limit. In the region $z>15$, this compensation is generally impossible, so the masses of such dwarfs cannot exceed this limit. The effects of GTR slightly reduce the mass of a dwarf, but cause the instability. The parameter $x_{0}^{*}$, at which instability occurs, does not depend on $z$, but only on $\tilde{\Omega}$. The critical value of $x_{0}^{*}$ decreases with increasing the parameter $\tilde{\Omega}$. The area of existence for helium, carbon, and oxygen depends on the effects of GTR. For magnesium, silicon, and iron, the instability is caused by the neutronization effects. This implies that, for the majority of real dwarfs, the processes of neutronization and the effects of GTR may be equally important.

Our conclusions are in accordance with the fact that the dwarfs with masses, which are very close or exceed Chandrasekhar's limit, are observed in binary systems, where the accretion plays a key role. After 
Table 9. Coefficients of Pade approximants in (68)

\begin{tabular}{|c|c|c|c|c|c|}
\hline Parameter & $a_{1}$ & $a_{2}$ & $a_{3}$ & $b_{1}$ & $b_{2}$ \\
\hline$\psi_{0,0}(\xi)$ & 0.153879 & 0.0053588 & 0.000783991 & 0.128076 \\
$A \psi_{0,2}(\xi)$ & -0.715078 & -0.0281033 & -0.00189924 & 0.246085 & 0.00472131 \\
$\psi_{1,0}(\xi)$ & -0.07444 & 0.00657361 & $-5.31141 \cdot 10^{-5}$ & 0.0325496 & $-8.49965 \times 10^{-5}$ \\
$\psi_{1,2}(\xi)$ & -0.0474332 & 0.220168 & -0.0139001 & 0.522588 & 0.000121221 \\
$\psi_{2,0}(\xi)$ & 0.0855804 & -0.0143799 & 0.000190155 & -0.00216296 & $4.69667 \times 10^{-5}$ \\
$\psi_{2,2}(\xi)$ & -0.399671 & 0.0584875 & -0.00113677 & 0.315363 & $0.02652 \times 10^{-5}$ \\
$y_{1}(\xi)$ & -0.499189 & -0.034939 & $-1.42124 \times 10^{-5}$ & 0.008952 \\
$y_{2}(\xi)$ & -0.377538 & 0.162672 & 0.000116145 & 0.057591 \\
\hline
\end{tabular}

all, the matter consisting of elements with small $z$ falls, during the accretion process, on a degenerate dwarf.

\section{APPENDIX 1.}

The equation of state in the electron-nuclear model at $T=0 \mathrm{~K}$

In work [13], the energy in the electron-nuclear model was presented in the form

$E(x \mid z)=E_{e}(x)+E_{\mathrm{pol}}(x \mid z)+E_{L}(x \mid z)$,

where $E_{e}(x)$ is the energy of the homogeneous relativistic electron subsystem with the Coulomb interactions, $E_{\mathrm{pol}}(x \mid z)$ is the energy of a polarization of the electron subsystem by nuclei, $E_{L}(x \mid z)$ is the energy of effective $n$-particle screening interparticle interactions.

Herewith,

$E_{e}(x)=E_{0}(x)+E_{\mathrm{HF}}(x)+E_{c}(x)$,

where $E_{0}(x)$ is the energy in the homogeneous ideal electron model, $E_{\mathrm{HF}}(x)=-3 /(4 \pi) N_{e} \alpha_{0} m_{0} c^{2} x$ is the Hartree-Fock approximation term, $E_{c}(x)==N_{e} m_{0} c^{2} \alpha_{0}^{2} \varepsilon_{c}(x)$ is the correlation energy, and $\alpha_{0}==e^{2} /(\hbar c)$ is the fine structure constant.

The dimensionless factor $\varepsilon_{c}(x)$ was approximated with the expression

$\varepsilon_{c}(x)=-\frac{b_{0}}{2} \int_{0}^{x} \frac{b_{1} a+t^{1 / 2}}{t^{3 / 2}+t b_{1} a+t^{1 / 2} b_{2} a^{2}+b_{3} a^{3}} \times$

$\times \frac{1+a_{1} t+a_{2} t^{2}}{1+d_{0} t} d t$

$a=\left(\alpha_{0} \eta\right)^{1 / 2} ; \eta=(9 \pi / 4)^{1 / 3} ; a_{1}=2.25328 ;$

$a_{2}=4.87991 ; d_{0}=0.924022 ; b_{0}=0.0621814 ;$

$b_{1}=9.81379 ; b_{2}=2.82214 ; b_{3}=0.69699$.

The polarization energy

$E_{\mathrm{pol}}(x)=N_{e} m_{0} c^{2} z \alpha_{0}^{3 / 2} \varepsilon_{\mathrm{pol}}(x)$,

where, in the approximation of two-particle correlations,

$\varepsilon_{\mathrm{pol}}(x)=-\int_{0}^{x} \frac{c_{0}+c_{1} t+c_{2} t^{2}+c_{3} t^{3}}{1+d_{1} t+d_{2} t^{2}+d_{3} t^{3}} d t$, $c_{0}=4.06151 ; c_{1}=32.6118 ; \quad c_{2}=-43.6587 ;$

$c_{3}=104.13 ; d_{1}=73.8252 ; d_{2}=-67.1028 ;$

$d_{3}=189.781$.

In the same approximation, the lattice energy (the sum of two-particle effective interactions)

$E_{L}(x \mid z)=N_{e} m_{0} c^{2} \alpha_{0} z^{0.61803} \varepsilon_{L}(x)$,

$\varepsilon_{L}(x)=-\int_{0}^{x} \frac{g_{1}+g_{2} t+g_{3} t^{2}}{1+q_{1} t+q_{2} t^{2}+q_{3} t^{3}} t d t$,

$g_{1}=18.5394 ; g_{2}=-15.7018 ; g_{3}=52.9999 ;$

$q_{1}=42.5037 ; q_{2}=-39.1122 ; g_{3}=132.253$.

The relationship between the pressure and the energy

$P(x \mid z)=\frac{d E(x \mid z)}{d V}=\frac{x^{4}}{N_{e}}\left(\frac{m_{0} c}{\hbar}\right)^{3} \frac{1}{9 \pi^{2}} \frac{d E(x \mid z)}{d V}$

leads to expression (6), where

$f(x \mid z)=2 \alpha_{0} x^{4}\left\{\frac{1}{\pi}-\right.$

$\left.-\frac{4}{3} \frac{d}{d x}\left(z^{0.61803} \varepsilon_{L}(x)+z \alpha_{0}^{1 / 2} \varepsilon_{\mathrm{pol}}(x)+\alpha_{0} \varepsilon_{c}(x)\right)\right\}$.

At large values of the relativistic parameter $x$, all energy terms are proportional to $x$. The contributions of three-particle correlations are negligible.

\section{APPENDIX 2.}

\section{Approximation for the basis functions}

The Pade approximant for $y_{0}(\xi)$ given in (31)

$f(\xi)=\frac{1+a_{1} \xi^{2}+a_{2} \xi^{4}+a_{3} \xi^{6}}{1+b_{1} \xi^{2}+b_{2} \xi^{4}+b_{3} \xi^{6}}$,

where

$a_{1}=37.9322, a_{2}=-0.339691, a_{3}=-0.00963653$,

$b_{1}=38.0974, b_{2}=5.9891, b_{3}=10.0382075$.

The Pade approximant for $y_{1}(\xi), y_{2}(\xi), \psi_{l, 0}(\xi), \psi_{l, 2}(\xi)$ (see (31))

$f(\xi)=\frac{a_{1} \xi^{2}+a_{2} \xi^{4}+a_{3} \xi^{6}}{1+b_{1} \xi^{2}+b_{2} \xi^{4}}$.

The coefficients are shown in Table 9 .

ISSN 2071-0194. Ukr. J. Phys. 2018. Vol. 63, No. 9 
1. W.S. Adams. The spectrum of the companion of Sirius. Publ. Astron. Soc. Pacific 27, 236 (1915).

2. R.H. Fowler. On dense matter. Mon. Not. R. Astron. Soc. 87, 114 (1926).

3. S. Chandrasekhar. The maximum mass of ideal white dwarfs. Astrophys. J. 74, 81 (1931).

4. S. Chandrasekhar. Stellar configurations with degenerate cores. Mon. Not. R. Astron. Soc. 95, 676 (1935).

5. E. Shatzman. The analysis of starlight: Two centuries of astronomical spectroscopy. Ann. Astrophys. 9, 144 (1946).

6. S.A. Kaplan. Superdense stars. Sci. Notes of I. Franko Lviv State Univ. Ser. Math. 4, 109 (1949).

7. R.A. James. The structure and stability of rotating gas masses. Astrophys. J. 140, 552 (1964).

8. E.E. Salpeter. Energy and pressure of a zero-temperature plasma. Astrophys. J. 134, 669 (1961).

9. Ya.B. Zeldovich, I.D. Novikov. Relativistic Astrophysics (Nauka, 1967) (in Russian).

10. S.L. Shapiro, S.A. Teukolsky. Black Holes, White Dwarfs and Neutron Stars (Cornell Univ., 1983).

11. M.V. Vavrukh, S.V. Smerechinskii. A finite-temperature Chandrasekhar model: Determining the parameters and calculation of the characteristics of degenerate dwarfs. Astronomy Reports 56 (5), 363 (2012).

12. M. Vavrukh, S. Smerechynskyi, D. Dzikovskyi. The influence of the axial rotation on the degenerate dwarfs characteristics. Mathematical Modeling and Computing 4 (1), 107 (2017).

13. M.V. Vavrukh, D.V. Dzikovskyi, N.L. Tyshko. Reference system approach within the white-dwarfs theory Condens. Matter Phys. 20 (4), 43001 (2017).
14. S. Chandrasekhar. The equilibrium of distorted polytropes. I. The rotational problem. Mon. Not. R. Astron. Soc. 93, 390 (1933).

15. A.H. Wapstra, K. Bos. The 1977 atomic mass evaluation: in four parts. Atomic Data and Nuclear Data Tables 19, 175 (1977).

16. T. Hamada, E. Salpeter. Models for zero-temperature stars. Europ. Astrophys. J. 133, 683 (1961).

Received 02.07.18

М.В. Ваврух, Д.В. Дзіковсъкий, С.В. Смеречинсъкий

ВРАХУВАННЯ КОНКУРУЮЧИХ

ФАКТОРІВ ПРИ РОЗРАХУНКАХ ХАРАКТЕРИСТИК НЕМАГНІТНИХ ВИРОДЖЕНИХ КАРЛИКІВ

Р е $з$ ю м е

На основі побудованого раніше рівняння стану електронядерної моделі при високих густинах та за допомогою рівняння механічної рівноваги досліджено вплив міжчастинкових взаємодій і осьового обертання на макроскопічні характеристики (маса, форма поверхні) масивних вироджених карликів. Запропоновано метод знаходження розв'язків рівняння рівноваги за наявності обертання, що грунтується на використанні базису універсальних функцій радіальної змінної. Встановлено умови, при яких осьове обертання може компенсувати зменшення маси, зумовлене кулонівськими взаємодіями. Визначено максимальне значення параметра релятивізму, при якому порушується стабільність за рахунок ефектів загальної теорії відносності. 